ORIGINAL ARTICLE

\title{
Bilateral asymmetry of pedaling force at different levels of exercise intensity in cycle ergometer
}

\author{
Lachezar G. Stefanov ${ }^{\mathrm{ABCDE}}$, Ivan Ivanov ${ }^{\mathrm{ABCDE}}$, Daniela Aleksieva ${ }^{\mathrm{ABCDE}}$ \\ National Sports Academy, Bulgaria
}

Authors' Contribution: A - Study design; B - Data collection; C - Statistical analysis; D - Manuscript; Preparation; E - Funds Collection.

\begin{abstract}
Purpose: $\quad$ To investigate how bilateral pedaling asymmetries change at exercises with different levels of intensity.

Material: $\quad$ Eight students of cycling, average age 25.4 years, were investigated. In the experiment, we recorded the pedal force of the right and left legs during three consecutive exercises of different intensity $35 \%, 55 \%$ and $85 \%$ respectively. To quantify the difference in physical parameters of pedaling between the left and right legs, we used two approaches that complement each other in the analysis of bilateral asymmetry. One approach involved determining an asymmetry index, and other was statistical analysis.

Results: $\quad$ The Student's t-test indicated that the difference between the power samples for the left and right pedals decreased at $85 \%$ exercise intensity vs. $55 \%$ with a statistical significance of $a=0.05$. The bilateral asymmetry in most cases decreased or in two cases remained unchanged. The cases where there was no statistically significant difference between the power samples for both legs at $85 \%$ and $55 \%$ intensity levels had the lowest asymmetry index.

Conclusions: $\quad$ The bilateral pedaling asymmetry is reduced at $85 \%$ exercise intensity compared to the $55 \%$ one. The reduction in asymmetry index ranges from $1 \%$ to $14.1 \%$, Combining the asymmetry index and the Student's t-test can increase the informativeness of pedaling power data when analyzing bilateral asymmetry.

Keywords: $\quad$ cycling, pedal force, bilateral asymmetry, asymmetry index, intensity.
\end{abstract}

\section{Introduction}

Asymmetry in morphology and function between the both sides of the body has long been known. It is also observed in everyday human activities. It is interesting to know how this asymmetry in cycling changes and the factors influence it.

It is well known that higher cycling power is closely linked to the ability to apply effective pedal force. There are several areas in which research could contribute to improving the understanding of the relationship between optimal force effectiveness and performance. Establishing a "natural" range of symmetry of pedal forces should be the goal of future research and may explain the influence of symmetry in cycling performance and injury prevention [1].

Focusing on a segment of the pedalling cycle may well offer improvement in performance [2, 3].

There are many studies on bilateral pedal differences that show some degree of asymmetry in force, torque, performance, and power between the two legs [4]. During cycling, bilateral differences are frequently found and vary with the competitive situation, pedaling cadence, exercise intensity and exercise duration [5]. It was concluded that pedaling asymmetry changes systematically with crank torque output and exercise intensity [6]. The results demonstrate that pedaling asymmetry in trained road cyclists can be consistent across different saddle heights and even at different cycling intensities [7]. Smak et al. conclude that pedaling asymmetry is highly variable

(c) Lachezar G. Stefanov, Ivan Ivanov, Daniela Aleksieva, 2020 doi:10.15561/26649837.2020.0507 among subjects and that individual subjects may exhibit different systematic changes in asymmetry with pedaling rate depending on the quantity of interest [8].

There are several types of forces acting on a cycle's crank. The total force applied perpendicularly to the crank of the pedal in the sagittal plane (the one in which the bicycle moves) consists of two components: normal force (direction and perpendicular to the surface of the pedal) and anterior-posterior force (it is perpendicular to the normal force). Effective force is a percentage of the total force and causes propulsion or stopping of the pedal movement [1].

There is a lot of investigation on the factors that influence the force applied to the pedals. The human ability to develop highest power values in a short time plays an important role in many sport disciplines and every day activity. Power depends on force and velocity [9]. The force-velocity and power-velocity characteristics in cycling typify the function of muscles responsible for pushing on the pedal, especially the hip and the knee extensors [10]. A procedure for decomposing pedal forces into muscular and nonmuscular components was proposed by Kautz S. and Hut M. to provide a theoretical basis for understanding or evaluating pedal forces [11]. Pedal force effectiveness is defined as the ratio between the effective force and the total force applied to the pedal [1].

The relationship between asymmetry and performance is greater for the effective pedaling power and less for total power and the performance index. Substantial asymmetry was found for effective force (36-54\%), resultant force 
(11-21\%), and the Index of effectiveness (21-32\%) [12].

Asymmetry in pedaling was found to be highly variable between subjects. They show different systematic changes depending on the speed of pedaling [8]. On the other hand, the increase in torque applied to the crank and the increase in the exercise intensity cause a decrease in the asymmetry of pedaling $[6,13]$. Another study shows that asymmetry in peak torque increases at higher load power and this is due to the dominant leg [14].

The quantitative assessment of asymmetry usually involves measuring the asymmetry index left-to-right or statistical procedures $[4,12]$. The index of bilateral asymmetry in pedaling varies widely, and the effect of cadence on it is not fully understood. There is agreement in the literature that bilateral differences in cycling power and performance can vary between $5 \%$ and $20 \%$ [5].

Of great importance for the asymmetry obtained in the various studies is the apparatus used. This is evidenced by the increase in torque differences for the dominant and non-dominant legs when increasing the applied power when different measurement systems are used. The crankmounted pressure-sensing system showed an asymmetry index of $7 \%$ to $33 \%$, and for the strain gauge system using tensometric pedals the index ranged from $9 \%$ to $66 \%$ [14].

The dominant leg is always responsible for the greater torque of the crank. As the torque increases and the exercise intensity increases, the asymmetry of pedaling decreases [6].

Therefore, we hypothesized how the magnitude of exercise intensity would affect asymmetry in pedaling.

The purpose of the study is to monitor how pedaling asymmetries change at exercises with three different levels of intensity, the latter of which should cause fatigue in participants. We chose a laboratory test because of the greater control over the experiment. In addition, laboratory maximum test data offer a precise way of modeling cycling performance [15].

\section{Material and methods}

Participants. Eight students, men, cyclists, average age 25.4 years, was investigated. All were assigned to the same experimental task. In this experiment, informed consent was obtained from all participants.

Procedure.

The investigation was accomplished in one session for each participant. The preferred leg was pre-determined by a "kick to the target" test $[16,17]$. The subjects were instructed to measure their resting heart rate after awakening from sleep (HRrest) and to report it to the researchers. This was necessary to determine the exercise intensity. The hardware complex we used in this study consisted of a Monark 618-E bicycle ergometer, a Sigma - Sport heart rate monitor, a laptop, Wi-Fi instrumented pedals, fixed to the bicycle ergometer._The system also includes an interface, created and described by Stefanov [18]. The system measures the normal force. Every one of the students monitored used their own cycling shoes SPDSL pedal system designed by Shimano. Prior to the test, the maximum heart rate (HRmax) of each participant was theoretically determined according to the Tanaka formula [19].

$$
\text { HRmax }=208-0.7 x \text { age }
$$

The target heart rate (THR), which should be reached and maintained during the three intensity levels, was calculated as $35 \%, 55 \%$ and $85 \%$ of the reserve heart rate (HRR), respectively. We used the Karvonen formula to determine the target heart rate:

$$
\text { THR }=(\text { HRR } x(\% \text { of intensity }))+\text { HRrest }
$$

where HRR $=$ HRmax - HRrest.

After this preparation three consecutive exercise intensity are set without any rest between them with the following levels of intensity and duration:

- Level I - 35\% of HRR, which is 5\% lower than the upper boundary of the area designated as light intensity by [20]. After reaching the THR, the investigated person worked for 4 minutes at such intensity as to keep the heart rate constant. This level plays the role of warm-up.

- Level II - 55\% of HRR, which is 5\% lower than the upper boundary of the area defined as moderate intensity by [20]. After reaching the THR, the investigated worked for 10 minutes with such intensity, that they kept the heart rate constant.

- Level III - $85 \%$ of HRR, which is defined as a very high intensity area according to [20]. After reaching the THR, the tester works until he can maintain a constant heart rate and pedal cadence or until a subjective feeling of fatigue occurs.

The measurements of the instrumented pedals were monitored by the researcher team throughout the experiment, but recorded in the last 30 seconds on the first two levels of the test to ensure that a steady-state was reached. On the last level longer periods of time were recorded in order to reach the moment of fatigue. The cool-down was then performed at an intensity of about $30 \%$ of HRR for $4 \mathrm{~min}$, which ended the experiment.

Throughout the experiment, the subject had to maintain a pedaling cadence of 60 revolutions per minute. At this frequency, we expect a lower error in the reported power [21]. In addition, as pedaling speed increases, asymmetry decreases [8], and we were interested in its manifestation.

During the experiment, we recorded the pedal force of the legs on the left and right pedals every $100 \mathrm{~ms}$ of the pedal trajectory. At a cadence of $60 \mathrm{rpm}$ we received 5 measurements for each left and right leg respectively, for each revolution of the pedal. In fact, with this cadence, we get 150 measurements for the left and right pedals in 30 seconds. These measurements were transmitted wirelessly to the laptop. An EXCEL application inserts data directly into the spreadsheet. After the experiment, the inserted data were processed with macros created for the purposes of the study. The instrumented pedal system wirelessly registered and transmitted the force applied on them. 
The work done for angular movement (movement angle) can be expressed by the formula:

$$
\mathrm{W}=\mathrm{F} \theta \mathrm{r}
$$

where: $\mathrm{W}=\operatorname{work}($ in $\mathrm{J}) ; \mathrm{F}=$ force $($ in $\mathrm{N}) ; \theta=\operatorname{angle}($ in $\mathrm{rad}) ; \mathrm{r}=\operatorname{radius}(\mathrm{m})$

At $60 \mathrm{rpm}, \theta=0.628, \mathrm{r}=$ radius $(\mathrm{m})$ the length of the crank is $0.165 \mathrm{~m}$

The power $(\mathrm{P})$ with which each leg rotates the pedal for these $100 \mathrm{~ms}(\Delta \mathrm{t})$ is:

$$
\mathrm{P}=\mathrm{W} / \Delta \mathrm{t}
$$

Statistical analysis.

In the statistical analysis we used the EXCEL program of Microsoft Office Professional Plus 2010, and the statistical program is XLSTAT 2015.

After checking the shape of distribution of the samples by the Shapiro-Wilk normality test. This statistical method stated:

- The null hypothesis H0: The variable from which the sample was extracted follows a Normal distribution; and the alternative hypothesis Ha: The variable from which the sample was extracted does not follow a Normal distribution.

- As the computed p-value is greater than the significance level alpha $=0.05$, one cannot reject the null hypothesis $\mathrm{H} 0$.

The results of the normality test are presented in Table 1. Represents the p-values from Shapiro-Wilk normality test for the peak power outputs for the left and right legs at an intensity of $35 \%((\mathrm{~L} \ell / \mathrm{P})$ and $(\mathrm{R} \ell / \mathrm{P})), 55 \%((\mathrm{Lm} / \mathrm{P})$ and $(\mathrm{Rm} / \mathrm{P}))$, and $85 \%((\mathrm{Lh} / \mathrm{P})$ and $(\mathrm{Rh} / \mathrm{P}))$ of HRR. We found that in most of samples the distribution is normal or close to normal, but in tree samples is not normal. Therefore, we chose nonparametric methods for further statistical processing.

We also use Kruskal-Wallis test (comparison of $\mathrm{k}$ samples).

\section{Results}

After initially transformation of the received variables into work (W) and then into power (P), we transferred only the peak power for each revolution of pedals for the left and right leg into Table 1 . Thus the peak power formed statistical samples for the left and right leg at intensities of $35 \%$ of HRR, $55 \%$ of HRR, and $85 \%$ of HRR described in the methodology.

To quantify the difference in physical parameters of pedaling between left and right leg, we used two approaches that complement each other in the analysis of bilateral asymmetry.

One approach involved determining an asymmetry index (AI), that has been described by different authors, with some differences in the equations due to the differences in the experiments in which they used it. We have chosen the equation for AI used by [6] to study bilateral asymmetry in cyclists.

$$
\mathrm{AI} \%=[(\mathrm{DO}-\mathrm{ND}) / \mathrm{DO}] 100,
$$

where: $\mathrm{AI} \%$ is an asymmetry index; $\mathrm{DO}$ is the measurement of the dominant leg; ND is the measurement from the nondominant leg. Two of the participants showed dominance for the left leg, which we took into account, when using the equation.

The other approach is statistical. We compared the absolute difference between peak power samples for the left and right pedals at 55\% HRR and $85 \%$ HRR intensity by Kruskal-Wallis test (comparison of $\mathrm{k}$ samples). We used a statistical significance $\alpha=0.05$. If $\mathrm{p}<0.05$ the two samples differed significantly from each other, and therefore the medians derived from them would also differ statistically significantly.

Presented medians (Tabl. 2) are obtained from the samples with peak power during each revolution of pedals for the left or right leg with one of the three exercise intensities. Figure 1. graphically presents the left and right leg power of one of the participants on the level of $85 \%$

Table 1. Shapiro-Wilk normality test p-values for the peak power outputs for the left and right legs.

\begin{tabular}{lllllll}
\hline Participants & $\mathbf{( L \ell / P )}$ & $\mathbf{( R \ell / P )}$ & $\mathbf{( L m / P )}$ & $\mathbf{( R m / P )}$ & (Lh/P) & (Rh/P) \\
\hline VA & 0.707897 & 0.108736 & 0.893029 & 0.68002 & 0.141679 & 0.347692 \\
DA & 0.864262 & 0.005072 & 0.598636 & 0.299225 & 0.091959 & 0.066211 \\
SS & 0.294762 & 0.536652 & 0.396569 & 0.757072 & 0.550043 & 0.084405 \\
DS & 0.033551 & 0.703211 & 0.963399 & 0.556498 & 0.639237 & 0.616726 \\
SH & 0.363315 & 0.890008 & 0.778219 & 0.748239 & 0.266807 & 0.661179 \\
MV & 0.619129 & 0.240705 & 0.148447 & 0.214255 & 0.242388 & 0.728578 \\
SI & 0.654552 & 0.610454 & 0.47261 & 0.748396 & 0.171393 & $<0.0001$ \\
SR & 0.054444 & 0.485225 & 0.97837 & 0.18201 & 0.356649 & 0.156173 \\
\hline
\end{tabular}

NOTE: Le/P - p-values for the peak power output for the left leg at intensities of 35\% of HRR; Rl/P - $p$-values for the peak power output for the right leg at intensities of 35\% of HRR; Lm/P - p-values for the peak power output for the left leg at intensities of 55\% of HRR; Rm/P - p-values for the peak power output for the right leg at intensities of $55 \%$ of HRR; Lh/P - p-values for the peak power output for the left leg at intensities of $85 \%$ of HRR; Rh/P - $p$-values for the peak power output for the right leg at intensities of $85 \%$ of HRR. 
intensity of HRR recorded every $100 \mathrm{~ms}$. You can also see the peaks for each revolution of the left and right leg pedals. The left and right leg peaks form the samples we compared.

Table 3 shows the average differences between left right legs in peak power at moderate to very high intensity ( $\mathrm{Lm} / \mathrm{Rm}_{\text {diff }}$ and $\mathrm{Lh} / \mathrm{Rh}_{\text {diff }}$ ), because those at low intensity $\left(\mathrm{L} \ell_{\text {diff }} / \mathrm{R} \ell_{\text {diff }}\right)$ show uncharacteristic changes. At moderate intensity, most participants showed a statistically significant average difference between peak power for the left and right legs, indicating the presence of bilateral asymmetry in pedaling. At $85 \%$ intensity, ( $p$-value above 0.05 ) the average difference between the peak power values for the left and right legs is not statistically significant. Therefore, bilateral asymmetry cannot be demonstrated in very high-intensity pedaling.

Based on both approaches, we made an individual analysis for each participant.

VA - The absolute difference between the medians of the peak power samples of the two legs at an intensity of $85 \%$ is greater than the one at the intensity of $55 \%$, but the samples from which these peak capacities come do not differ from each other with sufficient statistical significance. AI in both conditions changed slightly ( $1 \%$ for the right leg and $1.1 \%$ for the left leg), as AI for very high intensity was $(-1.1 \%)$. Therefore, the asymmetry does not change significantly.

SS - The absolute difference at both intensities is similar, but only the difference between the two legs for a $55 \%$ intensity is statistically significant, compared to $85 \%$. The AI for the intensity of $85 \%$ is less than that for the moderate intensity by $6.6 \%$. Asymmetry therefore decreases.

MV - The absolute difference is increasing, albeit slightly, at an intensity of $85 \%$ compared to that of $55 \%$. The statistical significance of the difference between the two peak power samples at both intensities is high. AI though slightly (3\%) decreases. Asymmetry therefore decreases.

DS - The absolute difference increases slightly at an intensity of $85 \%$ compared to that of $55 \%$. There was a statistically significant difference between the two

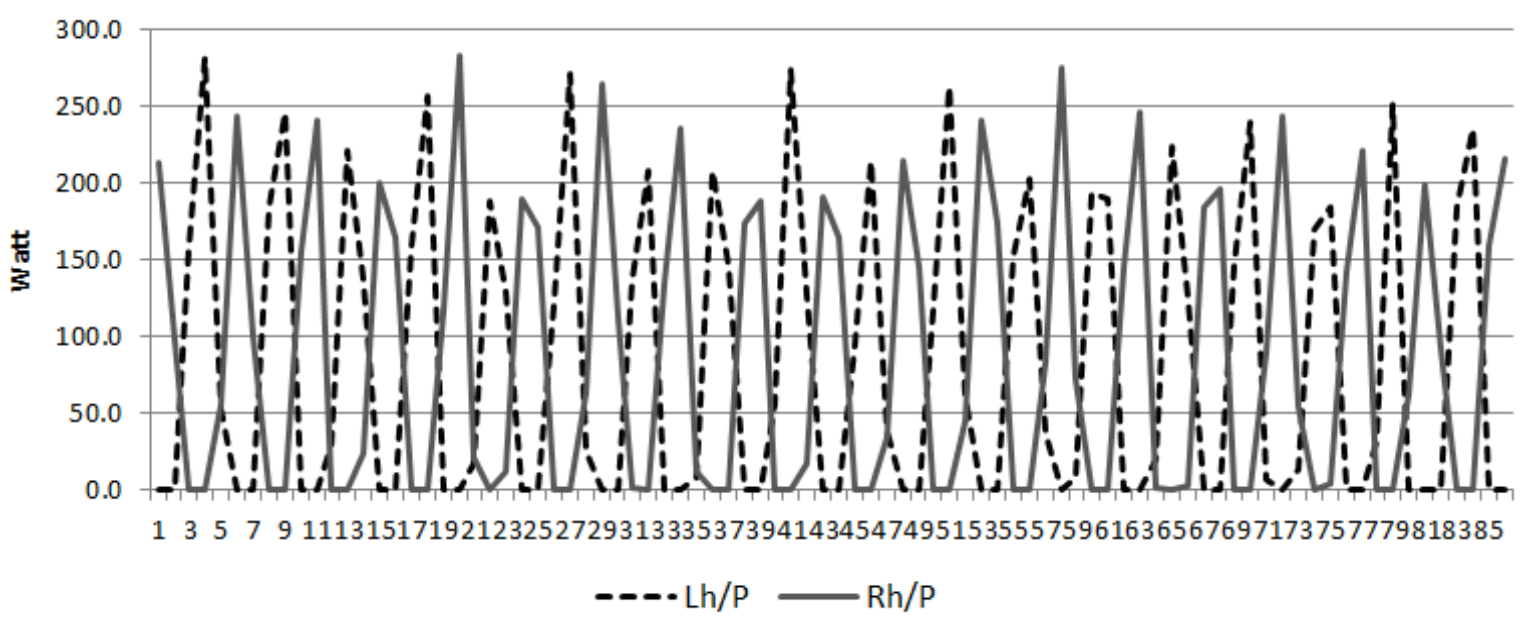

Figure 1. Represents the powers for left Lh / P and right Rh / P leg at an intensity of $85 \%$ of HRR. The ordinate shows the power of each measurement and abscissa the number of measurement.

Table 2. Peak power for left and right legs.

\begin{tabular}{|c|c|c|c|c|c|c|}
\hline Participants & $\begin{array}{l}\text { Le/P } \\
\text { (Watt) }\end{array}$ & $\begin{array}{l}\text { Re/P } \\
\text { (Watt) }\end{array}$ & $\begin{array}{l}\text { Lm/P } \\
\text { (Watt) }\end{array}$ & $\begin{array}{l}\text { Rm/P } \\
\text { (Watt) }\end{array}$ & $\begin{array}{l}\text { Lh/P } \\
\text { (Watt) }\end{array}$ & $\begin{array}{l}\text { Rh/P } \\
\text { (Watt) }\end{array}$ \\
\hline VA & 213.8 & 218.7 & 235.3 & 237.7 & 318.95 & 315.55 \\
\hline SS & 208.7 & 204.55 & 158.95 & 180.75 & 236.95 & 253.85 \\
\hline MV & 171.4 & 178.2 & 223.8 & 271.9 & 296.1 & 346.55 \\
\hline DS & 182.85 & 203.6 & 230.6 & 218.1 & 340.1 & 332.6 \\
\hline SR & 108.9 & 127.6 & 144.2 & 177.9 & 223.8 & 235.2 \\
\hline $\mathrm{SI}$ & 178.1 & 201 & 238 & 263 & 554.25 & 604.6 \\
\hline DA & 188.9 & 192.8 & 262.2 & 259.9 & 180.8 & 175.7 \\
\hline $\mathrm{SH}$ & 142.85 & 115.3 & 151.9 & 125.45 & 285 & 247.8 \\
\hline
\end{tabular}

NOTE: Le / P - low intensity, left leg peak power; Re / P - low intensity, right leg peak power; Lm / P - moderate intensity, left leg peak power; Rm / P - moderate intensity, right leg peak power; Lh / P-very high intensity, left leg peak power; Rh / $\mathrm{P}$ - very high intensity, right leg peak power. 
Table 3. Statistical significance in peak power difference between left and right legs, and Al for moderate and high intensity.

\begin{tabular}{|c|c|c|c|c|c|c|}
\hline Participants & $\mathrm{Lm} / \mathrm{Rm}_{\text {diff }}$ & $\mathrm{Lh} / \mathrm{Rh}_{\text {diff }}$ & $\begin{array}{l}\mathrm{Lm} / \mathrm{Rm} \\
\mathrm{p} \text { - value }\end{array}$ & $\begin{array}{l}\text { Lh/Rh } \\
p \text { - value }\end{array}$ & $\mathrm{Al} / \mathrm{m} \%$ & $A l / h \%$ \\
\hline VA & 7.4 & 31.25 & 0.74 & 0.0995 & 1.0 & -1.1 \\
\hline SS & 21.5 & 20.55 & $<0.0001$ & 0.29 & 13.4 & 6.8 \\
\hline MV & 40.3 & 42.8 & $<0.0001$ & $<0.0001$ & 17.7 & 14.6 \\
\hline DS & 19.2 & 20.4 & 0.048 & 0.31 & 6.5 & 1.1 \\
\hline$S R$ & 33.1 & 25.8 & $<0.0001$ & 0.755 & 18.9 & 4.8 \\
\hline SI & 30 & 50.35 & 0.028 & 0.083 & 9.5 & 8.3 \\
\hline DA & 24.3 & 32.6 & 0.91 & 0.69 & 0.9 & 2.8 \\
\hline SH & 20.75 & 25.8 & 0.001 & 0.000 & 17.4 & 13.1 \\
\hline
\end{tabular}

NOTE: $\mathrm{Lm} / \mathrm{Rm}_{\text {diff }}$ - differences in peak power at moderate intensity between left and right legs. $\mathrm{Lh}_{\text {/ }} \mathrm{Rh}_{\text {diff }}$ - differences in peak power at high intensity between left and right legs. Lm / Rm - statistical significance of the difference between the peak power outputs for left and right leg, at moderate intensity. Lh / Rh - statistical significance of the difference between the peak power outputs for left and right leg, at high intensity. Al / m\% - asymmetry index at moderate intensity; Al / h\% - asymmetry index at very high intensity.

samples only at moderate intensity, indicating that an $85 \%$ intensity difference may be apparent. The very lack of a statistically significant difference between the left and right leg samples at an intensity of $85 \%$ indicates little asymmetry. AI decreased by $5.4 \%$ at high intensity. Asymmetry therefore decreases.

SR - The absolute difference in high intensity decreases noticeably, but only the difference between the two peak intensity samples at moderate intensity is statistically significant. AI decreased by $14.1 \%$. Asymmetry therefore decreases.

SI - The absolute intensity difference of $85 \%$ is greater than that of $55 \%$, but only the one between the two $55 \%$ intensity samples is statistically significant. AI declines relatively slightly by $1.2 \%$. Asymmetry therefore decreases slightly.

DA - The absolute difference in high intensity is greater than that of moderate intensity, but the samples from which these differences come do not differ significantly. As AI increases slightly (1.9\%), asymmetry cannot be assumed to change.

SH - The absolute differences at both intensities are large, but the difference between them is relatively small. The statistical significance of the difference between the peak power extracts for the left and right legs at both intensities is high. AI decreased by $4.3 \%$. Asymmetry therefore decreases.

If we summarize the analyses for each participant, it can be seen that the bilateral asymmetry in most cases decreases but in two cases remains unchanged. The cases where there is no statistically significant difference between peak power samples for both legs at both intensity levels have the lowest AI.

\section{Discussion}

The reduction of bilateral asymmetry with increasing exercise intensity for each of the participants in the experiment was manifested to a different extent. Contingents studied by other authors are similar to the contingent studied by us, with asymmetry reaching $43 \%$ $[8,12]$.

The reduction in AI ranges from $1 \%$ to $14.1 \%$, which is similar to the conventional limit from $5 \%$ to $20 \%$ [5]. These differences in the degree of bilateral asymmetry depend on many factors. One of them is probably the degree of fatigue achieved in each participant. The level of $85 \%$ from HRR was intended to cause fatigue, which during the experiment we tried to detect on an external basis, such as inability to maintain pedaling speed, impaired pedaling, heart rate above target, which we defined and the subjective feeling of the participant. From this point of view, the degree of fatigue achieved for each cyclist is different.

Probably important for the degree of bilateral asymmetry is the functional condition of the participant as well as his pedaling technique. According to the preliminary information we received for each of them, those with more experience and higher achievement had lower AI or the difference of AI between the two levels of intensity was smaller.

Reduction of asymmetry of pedaling at high exercise intensity probably depends on the peripheral and central nervous mechanisms. Asymmetry in pedaling seems to be related to the dominance of the legs and is significantly reduced with increasing of exercise intensity. The effect of experience on asymmetry in pedaling is not as clearly defined as it is with asymmetry in running [5]. Future studies on bilateral asymmetry in pedaling should include 
larger and more homogeneous contingents from athletes. Standardized exercises similar to those used by us should be applied. We recommend using the complementary approach we use to evaluate bilateral asymmetry.

\section{Conclusions}

In conclusion, bilateral pedal asymmetry is reduced at high exercise intensity compared to moderate exercise intensity. Combining the AI and Student's t-test can increase the informativeness of pedaling power data when analyzing bilateral asymmetry. The lack of statistical significance for the difference between the left and right peak power samples in itself implies also less bilateral asymmetry.

\section{Conflict of interest}

The authors declare that there is no conflict of interests.

\section{References}

1. Bini R, Hume P, Crof J, Kilding A. Pedal force effectiveness in cycling: A review of constraints and training effects. Journal of Science and Cycling. 2013;2:11-24.

2. Sanderson DJ, Black A. The effect of prolonged cycling on pedal forces. Journal of Sports Sciences, 2003;21:191-9. https://doi.org/10.1080/0264041031000071010

3. Too D. Biomechanics of Cycling and Factors Affecting Performance. Sports Medicine, 1990;10:286-302. https://doi.org/10.2165/00007256-199010050-00002

4. Bini RR, Jacques TC, Sperb CH, Lanferdini FJ, Vaz MA. Pedal force asymmetries and performance during a $20-\mathrm{km}$ cycling time trial. Kinesiology (Zagreb, Online), 2016;48:193-9. https://doi.org/10.26582/k.48.2.12

5. Carpes FP, Mota CB, Faria IE. On the bilateral asymmetry during running and cycling - A review considering leg preference. Physical Therapy in Sport, 2010;11:136-42. https://doi.org/10.1016/j.ptsp.2010.06.005

6. Carpes FP, Rossato M, Mota CB, Faria IE. Bilateral Pedaling Asymmetry during a Simulated $40 \mathrm{~km}$ Cycling Time -Trial: 2206. Medicine \& Science in Sports \& Exercise, 2006;38:S394. https://doi.org/10.1249/00005768-200605001-02540

7. Diefenthaeler F, Berneira JDO, Moro VL, Carpes FP. Influence of saddle height and exercise intensity on pedalling asymmetries in cyclists. Rev Bras Cineantropom Desempenho Hum, 2016;18:411. https://doi.org/10.5007/1980-0037.2016v18n4p411

8. Smak W, Neptune RR, Hull ML. The influence of pedaling rate on bilateral asymmetry in cycling. Journal of Biomechanics, 1999;32:899-906. https://doi.org/10.1016/S0021-9290(99)00090-1

9. Busko K. Power output and mechanical efficiency of human muscle in maximal cycle ergometer efforts at different pedalling rates. Biology of Sport. 2005;22: 35-51.

10.Dorel S, Hautier CA, Rambaud O, Rouffet D, Van Praagh E, Lacour J-R, et al. Torque and Power-Velocity Relationships in Cycling: Relevance to Track Sprint Performance in World-Class Cyclists. Int J Sports Med, 2005;26:739-46. https://doi.org/10.1055/s-2004-830493

11.Kautz SA, Hull ML. A theoretical basis for interpreting the force applied to the pedal in cycling. Journal of Biomechanics, 1993;26:155-65. https://doi.org/10.1016/0021-9290(93)90046-H

12.Bini R. Relationship between pedal force asymmetry and performance in cycling time trial. J. Sports Med. Phys. Fitness. 2015;55: 1-2.

13.Rannama I, Port K. Bilateral Biomechanical Asymmetry During 30 Seconds Isokinetic Sprint-Cycling Exercise. LASE Journal of Sport Science, 2015;6:1-14. https://doi.org/10.1515/ljss-2016-0001

14.Bini RR, Hume PA. Assessment of Bilateral Asymmetry in Cycling Using a Commercial Instrumented Crank System and Instrumented Pedals. International Journal of Sports Physiology and Performance, 2014;9:876-81. https://doi.org/10.1123/ijspp.2013-0494

15.Gardner AS, Martin JC, Martin DT, Barras M, Jenkins DG. Maximal torque- and power-pedaling rate relationships for elite sprint cyclists in laboratory and field tests. Eur J Appl Physiol, 2007;101:287-92. https://doi.org/10.1007/s00421-007-0498-4

16.Liu T, Jensen JL. Age-Related Differences in Bilateral Asymmetry in Cycling Performance. Research Quarterly for Exercise and Sport, 2012;83:114-9. https://doi.org/10.1080/02701367.2012.10599832

17.van Melick N, Meddeler BM, Hoogeboom TJ, Nijhuis-van der Sanden MWG, van Cingel REH. How to determine leg dominance:Theagreementbetweenself-reportedandobserved performance in healthy adults. PLOSONE, 2017;12:e189876. https://doi.org/10.1371/journal.pone.0189876

18.Stefanov LG. Wireless cycle power-meter system with load cell. International Journal of Scientific \& Engineering Research, 2016;7, 805-809.

19. Tanaka H, Monahan KD, Seals DR. Age-predicted maximal heart rate revisited. Journal of the American College of Cardiology, 2001;37:153-6. https://doi.org/10.1016/S0735-1097(00)01054-8

20.Pollock ML, Gaesser GA, Butcher JD, Després J-P, Dishman RK, Franklin BA, et al. ACSM Position Stand: The Recommended Quantity and Quality of Exercise for Developing and Maintaining Cardiorespiratory and Muscular Fitness, and Flexibility in Healthy Adults. Medicine \& Science in Sports \& Exercise, 1998;30:975-91. https://doi.org/10.1249/00005768-199806000-00032

21.Pallarés JG, Lillo-Bevia JR. Validity and Reliability of the PowerTap P1 Pedals Power Meter. Journal of Sports Science and Medicine, 2018;17, 305-311. 


\section{Information about the authors:}

Lachezar G. Stefanov; (Corresponding Author); M.D., Ph.D., Associate Professor; http://orcid.org/0000-0001-5380-3446; luchos@nsa.bg; Department of Physiology and Biochemistry, National Sports Academy; Sofia, Bulgaria.

Ivan Ivanov; Ph.D., Associate Professor; http://orcid.org/0000-0002-0402-2894; kolev_nsa@abv.bg; Department of Technical and Ice Sports, National Sports Academy, Sofia, Bulgaria.

Daniela Aleksieva; M.D., Ph.D., Assistant; http://orcid.org/ 0000-0002-1476-3063; aleksieva.daniela@gmail.com; Department of Physiology and Biochemistry, National Sports Academy; Sofia, Bulgaria.

Cite this article as:

Stefanov L, Ivanov I, Aleksieva D. Bilateral asymmetry of pedaling force at different levels of exercise intensity in cycle ergometer. Pedagogy of physical culture and sports, 2020;24(5):264-270.

https://doi.org/10.15561/26649837.2020.0507

This is an Open Access article distributed under the terms of the Creative Commons Attribution License, which permits unrestricted use, distribution, and reproduction in any medium, provided the original work is properly cited (http://creativecommons.org/licenses/by/4.0/deed.en).

Received: 18.03.2020

Accepted: 30.04.2020; Published: 30.10.2020 